\title{
Oligopolistic Competition of Gencos in Reactive Power Ancillary Service Provisions
}

\author{
Puneet Chitkara, Member, IEEE, Jin Zhong, Member, IEEE, and Kankar Bhattacharya, Senior Member, IEEE
}

\begin{abstract}
The need for a market based approach for reactive power procurement has been recognized by FERC. Due to the localized characteristic of reactive power, regulatory mechanisms are necessary to mitigate the possibility of holding market power by generators. The regulator is responsible for devising the regulatory mechanisms that provide incentives to generators to supply reactive power while preventing the abuse of market power. This paper proposes a mathematical model to simulate the strategic behavior of generators supplying reactive power while considering the system operator's schedule. The proposed method can be used by the regulator to simulate the market behavior in the reactive power supply. We further study how regulatory policies affect the strategic behavior. An alternative price cap method has been proposed and tested in the numerical example to mitigate the effect of strategic behavior of generators in reactive power procurement by the system operator. The numerical examples are tested on the Nordic 32-bus system.
\end{abstract}

Index Terms-Mathematical problem with complementarity constraints, multi-leader-follower games, reactive power, strategic offering, supply function equilibrium.

\section{NOMENCLATURE:}

Indices:

$f \quad$ Index for gencos owning generating units.

$\mathrm{H}_{\mathrm{f}} \quad$ Set of all generators belonging to genco $f$.

$\mathrm{N} \quad$ Total number of buses in the system.

F Total number of gencos in the market.

Variables:

$\mathrm{P}_{\mathrm{fi}} \quad$ Active power generation from a generator owned by genco $f$ at bus $i$, p.u.

$\mathrm{Q}_{\mathrm{fi}} \quad$ Reactive power generation from a generator owned by genco $f$ at bus $i$, p.u.

$\mathrm{V}_{\mathrm{i}} \quad$ Voltage magnitude at bus $i$, p.u.

$\theta_{i} \quad$ Voltage angle at bus $i$, radians.

$\mathrm{k}_{\mathrm{fi}} \quad$ Strategic variable of generator belonging to genco $f$ at bus $i$.

Manuscript received October 16, 2008; revised February 05, 2009. Current version published July 22, 2009. This work was supported by the Research Grants Council of Hong Kong under Grant HKU 7196/07E. Paper no. TPWRS00821-2008.

P. Chitkara and J. Zhong are with the Department of Electrical and Electronic Engineering, University of Hong Kong, Hong Kong (e-mail: puneet.chitkara@gmail.com; jzhong@eee.hku.hk).

$\mathrm{K}$. Bhattacharya is with the Department of Electrical and Computer Engineering, University of Waterloo, Waterloo, ON N2L 3G1, Canada (e-mail: kankar@ece.uwaterloo.ca).

Digital Object Identifier 10.1109/TPWRS.2009.2023266 $\rho \quad$ Market clearing price of reactive power (\$/p.u. MVAr).

$\lambda^{P} \quad$ Dual of active power injection constraint.

$\lambda^{Q} \quad$ Dual of reactive power injection constraint.

$\delta^{\text {min }} \quad$ Dual of the minimum voltage limit constraint.

$\delta^{\max }$ Dual of the maximum voltage limit constraint.

$\beta^{\text {min }}$ Dual of minimum reactive power generation constraint.

$\beta^{\text {min }}$ Dual of maximum reactive power generation constraint.

$\alpha^{\text {min }}$ Dual of minimum active power generation constraint.

$\alpha^{\max }$ Dual of maximum active power generation constraint.

$\gamma \quad$ Dual of market clearing price determination constraint.

$\vec{K}_{f} \quad$ Vector of strategic variables of generators owned by genco $f$.

$\vec{w}_{f}^{I}=$

$\left[\lambda^{\rho \rho}, \delta 1^{\min }, \delta 1^{\max }, \beta 1^{\min }, \beta 1^{\max }, \gamma 1, \alpha 1^{\min }, \alpha 1^{\max }\right]$

Vector of dual variables attached to the inequality KKT conditions of ISO's problem.

$\vec{w}_{f}^{E}=$

$\left[\lambda^{o b j}, \lambda^{v}, \lambda^{P P}, \lambda^{\theta}, \lambda 1^{P}, \lambda 1^{Q}\right]$

Vector of dual variables attached to the equality

KKT conditions of ISO's problem.

$\vec{w}_{f}^{C}=$

$\left[\lambda^{\rho \rho 2}, \delta 2^{\min }, \delta 2^{\max }, \beta 2^{\min }, \beta 2^{\max }, \gamma 2, \alpha 2^{\min }, \alpha 2^{\max }\right]$

Vector of dual variables attached to complementarity KKT conditions of ISO's problem.

$\mu_{f}^{\max }$ Dual of maximum strategic variable constraint.

$\mu_{f}^{\min }$ Dual of minimum strategic variable constraint.

Constants:

$a_{f i}$

Intercept of generator's cost curve.

$b_{f i}$

Slope of generator's cost curve, \$/p.u. MVAr.

$V_{i}^{\min }$
Minimum voltage limit at each bus $i$, p.u. 


\begin{tabular}{|c|c|}
\hline$V_{i}^{\max }$ & $\begin{array}{l}\text { Maximum voltage limit at each } \\
\text { bus } i, \text { p.u. }\end{array}$ \\
\hline$Q_{f i}^{\min }$ & $\begin{array}{l}\text { Minimum reactive power } \\
\text { generation limit, p.u. }\end{array}$ \\
\hline$Q_{f i}^{\max }$ & $\begin{array}{l}\text { Maximum reactive power } \\
\text { generation limit, p.u. }\end{array}$ \\
\hline$P_{f i}^{C o n t}$ & Contract value of real power, p.u. \\
\hline$\Delta P_{f i}$ & $\begin{array}{l}\text { Variation allowed in contracted } \\
\text { real power, p.u. }\end{array}$ \\
\hline$P_{f i}^{\min }=P_{f i}^{c o n t}-\Delta P_{f i}$ & $\begin{array}{l}\text { Lower limit of real power } \\
\text { variation, p.u. }\end{array}$ \\
\hline$P_{f i}^{\max }=P_{f i}^{C o n t}+\Delta P_{f i}$ & $\begin{array}{l}\text { Upper limit of real power } \\
\text { variation, p.u. }\end{array}$ \\
\hline$k_{f i}^{\min }$ & $\begin{array}{l}\text { Minimum value of strategic } \\
\text { variable. }\end{array}$ \\
\hline$k_{f i}^{\max }$ & $\begin{array}{l}\text { Maximum value of strategic } \\
\text { variable. }\end{array}$ \\
\hline$P D_{i}$ & $\begin{array}{l}\text { Active power demand at bus } i \text {, } \\
\text { p.u. }\end{array}$ \\
\hline$Q D_{i}$ & $\begin{array}{l}\text { Reactive power demand at bus } i, \\
\text { p.u. }\end{array}$ \\
\hline$G_{i j}$ & $\begin{array}{l}\text { Element of the conductance } \\
\text { matrix, p.u. }\end{array}$ \\
\hline$B_{i j}$ & $\begin{array}{l}\text { Element of the susceptance } \\
\text { matrix, p.u. }\end{array}$ \\
\hline
\end{tabular}

\section{INTRODUCTION}

$\mathbf{R}$ EACTIVE power has been recognized as one of the ancillary services to be provided by generators for the reliable operation of the power system. It can also substantially improve the efficiency of delivering active power to customers. Furthermore, sufficient reactive power injected at certain locations can also alleviate transmission constraints and allow cheaper power to be delivered to heavy loaded areas. Because of the nature of reactive power supply, it needs to be procured and managed locally in an efficient and reliable manner. It has been recommended by FERC that generators providing reactive power be paid on a nondiscriminatory basis [1].

It has been argued in [2]-[4] that two components of payment be used for financial compensation to the generators providing reactive power ancillary services, 1) a capacity payment which is paid in advance for their readiness to produce/absorb reactive power, and 2) a usage component which is paid for reactive power actually dispatched in real-time. It is suggested in [2] that reactive power can be procured through long-term contracts between the independent system operator (ISO) and generators in order to isolate the reactive power prices from the impact of real-time market price volatilities. A competitive market framework for reactive power is proposed in [5]-[7] wherein a reactive power bidding structure is proposed and the reactive power provided by generators is settled at a uniform market price.
However, it is reported in [3], [6], and [7] that market power can exist in reactive power markets and that some reactive power suppliers can indeed manipulate reactive power market prices. In [8], the reactive market power is measured using must-run indices. It is noted in these studies that certain reactive power suppliers hold market power because of the limited number of supply-side providers at certain strategic buses coupled with the fact that transportation of reactive power over long distances is not a technically justifiable operating decision.

The market regulator is generally responsible for devising regulatory mechanisms that strike a balance between providing adequate incentives to generators to supply reactive power while also overseeing and preventing their abuse of market power.

In order to investigate the strategic behavior of generators, a reactive power procurement process and the strategic interactions between the ISO and generation companies (gencos) as well as amongst gencos is simulated in this paper.

In the literature on modeling strategic behavior in electricity markets, alternative representations have been used to model the conjectures of competing players. Hobbs [9] assumes Cournot conjectures between gencos in active power markets where generators sell power to the grid at the locational marginal price (LMP). Day et al. [10] use a conjectured supply function (CSF) model to demonstrate strategic behavior in active power markets. Wang et al. [11] also use a CSF approach to model an equilibrium problem with equilibrium constraints (EPEC) to determine the equilibrium strategies of various generators. The CSF model, as opposed to the Cournot model, allows the rivals to alter their supplies in response to price changes. All the above models use the dc load flow in their analysis.

Bautista et al. [12] analyze imperfect competition in active power markets using ac load flow model. The consideration of nonlinear ac load flow allows analysis of the impact of strategic behavior on reactive power and voltage constraints. The multi-leader-follower game presented in the paper has been formulated as a nonlinear programming (NLP) problem based on [13].

In this paper, a strategic bidding model considering ac load flow is presented that simulates the behavior of generators participating in reactive power procurement by the ISO. The game is formulated as a multi-leader-follower game. The ISO is assumed to be the follower, while the leaders are the gencos supplying reactive power. The gencos are assumed to have conjectures about the supply functions of their rivals; hence the solution represents the supply function equilibrium between gencos (leaders).

The proposed model can be used to analyze the strategic behavior of gencos in the system. Based on the results, some regulatory mechanisms are proposed that can restrict the effects of their strategic behavior. In the case studies, the effectiveness of the proposed alternative price cap regulation in alleviating abuse of market power is examined.

This paper is organized as follows: the mathematical model is formulated in Section II. The solution method is discussed in Section III. In Section IV, the model is tested on the Nordic 32-bus system. The results and discussions are presented. Section $\mathrm{V}$ concludes with suggestions for the regulatory policies on reactive power procurement. 


\section{MATHEMATICAL MODEL}

In this paper, the strategic behavior of generators providing for reactive power ancillary services is simulated with the assumption that the energy market has already been settled and that the active power from generators has already been scheduled. This assumption is in line with the reactive power management scheme proposed in [2] and [6]. However, in order to ensure that a feasible optimal solution is obtained, the active power production of each generator is allowed to vary within a narrow range $\Delta P_{f i}$ around the optimal dispatch $P_{f i}^{c o n t}$. In the reactive power optimization problem, the ISO minimizes the payment for reactive power and obtains the optimal reactive power dispatch. The gencos are assumed to offer their supply functions for reactive power strategically to maximize their profits. The reactive power market price is assumed to be uniform for all generators, and it is the offer price of the marginal generator which is selected for supplying reactive power.

In this paper the game is formulated as a multi-leader-follower game that has multiple dominant players (leaders)-the gencos and one follower-the ISO. Furthermore, the response of the follower (ISO) is constrained to be identical for each leader. In practical operation, the gencos are assumed to provide different quantities of reactive power at different offer prices. This means that the competition is more akin to supply function competition. The supply function submitted by each genco for each generator is $k_{f i}\left(a_{f i}+b_{f i} Q_{f i}\right)$, where $\left(a_{f i}+b_{f i} Q_{f i}\right)$ is the simplified linear marginal cost function of reactive power generation $Q_{f i}$ of the generator located at bus $i$ and belonging to genco $f$. Parameters $a_{f i}$ and $b_{f i}$ are the intercept and slope of the linear supply function of the generator belonging to genco $f$ at node $i$. In this paper, $k_{f i}$ is the strategic offer variable of each generator. The choice of strategic offer variable greater than unity allows the generator to offer reactive power price greater than the marginal cost. This variable is therefore an indicator of the extent to which each genco can exercise market power by charging a price greater that its marginal cost. Alternatively, $a_{f i}$ or $b_{f i}$ could have been chosen as the strategic variables [14]. The game between multi-gencos and the ISO is formulated in this paper as an EPEC. Such problems were introduced in [15] and further developed in [16] in the context of modeling competition in European electricity markets.

\section{A. ISO's Problem}

Given each generator's offer, the optimization problem faced by the ISO is to determine the reactive power output of each unit. The ISO minimizes the payment to be made to the generators for reactive power (1), subject to load flow (2)-(3), voltage limits (4)-(5), reactive power generation limits (6)-(7) and the constraint to ensure that the market price, for a given set of offers, is the highest reactive power offer accepted (10). The offer price of the genco is $k_{f i}\left(a_{f i}+b_{f i} Q_{f i}\right)$; therefore, $\rho$ in (10) determines the highest selected offer price from amongst all offers, which becomes the uniform market price for reactive power and is payable to all the gencos:

$$
\begin{aligned}
& \text { Maximize } J=-\sum_{f} \sum_{i} \rho Q_{f i} \text {. } \\
& \text { Subject to } \\
& \sum_{f} P_{f i}-P D_{i}=\sum_{j=1}^{N}\left|V_{i}\right|\left|V_{j}\right|\left(G_{i j} \cos \left(\theta_{i}-\theta_{j}\right)\right. \\
& \left.+B_{i j} \sin \left(\theta_{i}-\theta_{j}\right)\right) \quad \forall i \lambda_{i}^{P} \\
& \sum_{f} Q_{f i}-Q D_{i}=\sum_{j=1}^{N}\left|V_{i}\right|\left|V_{j}\right|\left(G_{i j} \sin \left(\theta_{i}-\theta_{j}\right)\right. \\
& \text { - } \left.B_{i j} \cos \left(\theta_{i}-\theta_{j}\right)\right) \forall i \lambda_{i}^{Q} \\
& -V_{i} \leq-V_{i}^{\min } \quad \forall i \quad \delta_{i}^{\min } \\
& V_{i} \leq V_{i}^{\max } \quad \forall i \quad \delta_{i}^{\max } \\
& -Q_{f i} \leq-Q_{f i}^{\min } \quad \forall i \quad \forall f \beta_{f i}^{\min } \\
& Q_{f i} \leq Q_{f i}^{\max } \quad \forall i \forall f \quad \beta_{f i}^{\max } \\
& -P_{f i} \leq-P_{f i}^{\min } \quad \forall i \forall f \quad \alpha_{f i}^{\min } \\
& P_{f i} \leq P_{f i}^{\max } \quad \forall i \forall f \alpha_{f i}^{\max } \\
& k_{f i}\left(a_{f i}+b_{f i} Q_{f i}\right) \leq \rho \quad \forall i \forall f \gamma_{f i} \\
& \rho \geq 0
\end{aligned}
$$

where the variables $\lambda_{i}^{P}, \lambda_{i}^{Q}, \delta_{i}^{\min }, \delta_{i}^{\max }, \beta_{f i}^{\min }, \beta_{f i}^{\max }, \alpha_{f i}^{\min }$, $\alpha_{f i}^{\max }$, and $\gamma_{f i}$ are the duals of the respective constraints. $\rho$ is the uniform market price for reactive power, which is the highest price of all accepted offers. $P_{f i}^{\min }=P_{f i}^{c o n t}-\Delta P_{f i}$ and $P_{f i}^{\max }=P_{f i}^{c o n t}+\Delta P_{f i}$, where $P_{f i}^{c o n t}$ is the contracted real power and $\Delta P_{f i}$ is the small variation allowed from contracted real power.

\section{B. Genco's Problem}

The gencos are required to offer price-quantity pairs in the reactive power auction. They are also aware that the reactive power demand is generally inelastic and hence these players would like to strategize their offers to maximize their profits from reactive power supply. The gencos need to decide how much reactive power and at what prices to supply in the market, since supply of reactive power could cause generator winding losses, as well as reduce/limit their cash flows from active power markets because of operational requirements in the opportunity region [2]-[7]. Hence the price offered for reactive power by a genco would be governed not only by the operation cost and opportunity cost but also the inelasticity of the reactive power demand. The genco's profit maximization problem is thus stated below, with the objective function $R_{f}$ denoting the profit of genco $f$ as

$$
\text { Maximize } R_{f}=\sum_{i} \rho Q_{f i}-\sum_{i} \int\left(a_{f i}+b_{f i} Q_{f i}\right) Q_{f i}
$$

Subject to

$$
\begin{array}{ll}
k_{f i}-k_{f i}^{\min } \geq 0 & \forall i \mu_{f i}^{\min } \\
k_{f i}^{\max }-k_{f i} \geq 0 & \forall i \mu_{f i}^{\max } .
\end{array}
$$

${ }^{1}$ This is a reasonable assumption since no genco is likely to supply at a price lower than its marginal cost. 
Equation (13) sets a lower bound on the strategic offer variable $k_{f i}$, and (14) sets an upper bound on it . For the purpose of analysis in this paper, it is assumed that the gencos do not bid below their marginal costs and hence the lower bound, $k_{f i}^{\min }$ is equal to unity for all gencos. ${ }^{1}$ The upper bound $k_{f i}^{\max }$ can be set by the gencos based on their experience in the market or based on the price cap set for reactive power procurement by the regulator.

While maximizing its profit, the genco takes into account the equilibrium conditions of the ISO (the follower). Hence, the Karush-Kuhn-Tucker (KKT) conditions of the ISO's problem are incorporated as constraints in the genco's problem. This will be a mathematical problem with equilibrium constraints (MPEC) for each genco. The extent to which a genco increases its offer price above its marginal cost is denoted by $k_{f i}$, which is a dimensionless quantity. Based on the past observation of the rival gencos in the market, each genco is also assumed to have an idea about the range in which the rival gencos can vary their $k_{f i}$.

\section{SOLUTION MeTHOD}

\section{A. Derivation of ISO's Optimality Conditions}

The KKT conditions (given in Appendix A) are necessary for the optimality of the ISO's optimization problem. Since the ISO's problem is not convex, the solution to the KKT conditions may lead to a saddle point or a local maximum. As pointed out in [15] and [16], this difficulty is inherent to nonconvex problems. It is suggested that a practical way to partially overcome this problem is to test different starting points.

Let $\mathbf{y}$ denote the vector of all decision variables and Lagrange multipliers of the ISO's problem, i.e.,

$$
\begin{aligned}
\mathbf{y}= & \left\{P_{f i}, Q_{f i}, V_{i}, \theta_{i}, \lambda_{i}^{Q}, \lambda_{i}^{P}, \delta_{i}^{\min }, \delta_{i}^{\max },\right. \\
& \left.\beta_{f i}^{\min }, \beta_{f i}^{\max }, \gamma_{f i}, \alpha_{f i}^{\min }, \alpha_{f i}^{\max }, \rho\right\} \\
= & \left\{\mathbf{y}^{\mathbf{0}}, \mathbf{y}^{\mathbf{1}}\right\}
\end{aligned}
$$

where $\mathbf{y}^{\mathbf{o}}=\left\{P_{f i}, Q_{f i}, V_{i}, \theta_{i}, \lambda_{i}^{Q}, \lambda_{i}^{P}\right\}$ and $\mathbf{y}^{\mathbf{1}}=$ $\left\{\delta_{i}^{\min }, \delta_{i}^{\max }, \beta_{f i}^{\min }, \beta_{f i}^{\max }, \gamma_{f i}, \alpha_{f i}^{\min }, \alpha_{f i}^{\max }, \rho\right\}$.

It may be noted that $\mathbf{y}^{\mathbf{0}}$ is a vector of free variables or variables within a predefined finite range, whereas $\mathbf{y}^{\mathbf{1}}$ is a vector of all variables constrained to be nonnegative. The KKT conditions of the ISO's optimization are parameterized in $\left\{\vec{K}_{1}, \ldots, \vec{K}_{f}, \ldots, \vec{K}_{F}\right\}$, where $F$ is the total number of gencos, and $\vec{K}_{f}=\left\{k_{f i}, \forall f i \in H_{f}\right\}$ denote the offer strategy of the generating company $f$. This is because the ISO has no control over the offer strategies of the gencos. The vector $\overrightarrow{z^{E}}\left(\vec{K}_{1}, \ldots, \vec{K}_{f}, \ldots, \vec{K}_{F}, \mathbf{y}\right)=0$ is used to represent all the equality KKT conditions of the ISO's problem given by (A1), (A2), (A3), (A4), (A7), and (A8) in Appendix A, and $\overrightarrow{z^{I}}\left(\vec{K}_{1}, \ldots, \vec{K}_{f}, \ldots, \vec{K}_{F}, \mathbf{y}\right) \geq 0$ to represent all the inequality KKT conditions of the ISO's problem given by (A5), (A9), (A11), (A13), (A15), (A17), (A19), and (A21), and $\overrightarrow{z^{C}}\left(\vec{K}_{1}, \ldots, \vec{K}_{f}, \ldots, \vec{K}_{F}, \mathbf{y}\right)=0$ to represent the complemen- tarity conditions given by (A6), (A10), (A12), (A14), (A16), (A18), (A20), and (A22).

\section{B. Formulation of MPEC for Each Genco}

The values of $\rho$ and $Q_{f i}$ in the genco's (leader's) problem are produced by the ISO's (follower's) optimality conditions (Appendix A). These variables can be expressed as the implicit functions of all genco's strategies $\left\{\vec{K}_{f}, f=1,2, \ldots, F\right\}$ and should satisfy KKT conditions of the ISO's optimization problem (Appendix A).

The optimization problem of each genco $f$ can then be described as the following MPEC (repeating (12)-(14) from above):

Maximize $R_{f}=\sum_{i} \rho Q_{f i}-\sum_{i} \int\left(a_{f i}+b_{f i} Q_{f i}\right) Q_{f i}$

Subject to

$$
\begin{aligned}
& k_{f i}-k_{f i}^{\min } \geq 0 \quad \forall i \mu_{f i}^{\min } \\
& k_{f i}^{\max }-k_{f i} \geq 0 \quad \forall i \mu_{f i}^{\max } \\
& \left.\vec{z}^{E}\left(\vec{K}_{1}, \ldots, \vec{K}_{f}, \ldots, \vec{K}_{F}, \mathbf{y}\right)=0 \quad \vec{w}_{f}^{E} \text { (dual vector }\right) \\
& \vec{z}^{I}\left(\vec{K}_{1}, \ldots, \vec{K}_{f}, \ldots, \vec{K}_{F}, \mathbf{y}\right) \geq 0 \quad \vec{w}_{f}^{I} \text { (dual vector) } \\
& \left.\vec{z}^{C}\left(\vec{K}_{1}, \ldots, \vec{K}_{f}, \ldots, \vec{K}_{F}, \mathbf{y}\right)=0 \quad \vec{w}_{f}^{C} \text { (dual vector }\right) \text {. }
\end{aligned}
$$

The incorporation of KKT conditions (15)-(17) reflects that each genco $f$ anticipates the impact of dispatch by the ISO on reactive power price and takes that into account in its decision making.

\section{Derivation of Genco's Optimality Conditions}

The Lagrangian of the genco's problem (18) is represented in terms of the first order conditions of the ISO's problem given in Appendix A. See equation (18) at the bottom of the next page.

The corresponding KKT conditions of each genco's optimization problem are derived as (19)-(30) at the bottom of the next page, where

$$
\begin{aligned}
& \vec{w}_{f}^{I}=\left\{\lambda^{\rho \rho}, \delta 1^{\min }, \delta 1^{\max }, \beta 1^{\min }, \beta 1^{\max }\right. \\
&\left.\gamma 1, \alpha 1^{\min }, \alpha 1^{\max }\right\} \\
& \vec{w}_{f}^{E}=\left\{\lambda^{o b j}, \lambda^{v}, \lambda^{P P}, \lambda^{\theta}, \lambda 1^{P}, \lambda 1^{Q}\right\} \text { and } \\
& \vec{w}_{f}^{C}=\left\{\lambda^{\rho \rho 2}, \delta 2^{\min }, \delta 2^{\max }, \beta 2^{\min }, \beta 2^{\max }\right. \\
&\left.\gamma 2, \alpha 2^{\min }, \alpha 2^{\max }\right\} .
\end{aligned}
$$

$\left(\vec{w}_{f}^{I}, \vec{w}_{f}^{E}, \vec{w}_{f}^{c}\right)$ are the vectors consisting of Lagrange multipliers of the KKT conditions of the ISO's problem appended to the genco's problem. It is assumed that these variables are same for each leader (genco), i.e., each genco prices the shared set of constraints arising from the ISO in a similar manner.

\section{Formulation of Equilibrium Between Gencos}

The Nash equilibrium solution is computed by solving (19)-(30) simultaneously for all the gencos. This leads to the 
formulation of an EPEC. Therefore, if $k_{f i}^{*}$ is the optimal strategy of genco $f$ at node $i$, provided that the strategies of other gencos are also optimal responses, then $k_{f i}^{*}$ must satisfy the KKT conditions of the optimization problems of all gencos. To put it in a vector notation, the equilibrium $\left(\vec{K}_{1}^{*}, \ldots, \vec{K}_{f}^{*}, \ldots, \vec{K}_{F}^{*}\right)$ must satisfy the KKT conditions of the optimization problem of each genco. That is, the equilibrium can be derived from the solution of the problem formed by combining the KKT conditions of all gencos' optimization problems. This however leads to a nonsquare nonlinear complementarity problem (NCP).

\section{E. Solving EPEC as a Single NLP}

A square NCP requires that all equation-variable pairs be uniquely matched. In (18), we find that the complementarity conditions of the ISO's problem are associated with dual variables $\vec{w}_{f}^{C}$. For example, the derivative of Lagrangian (18) with respect to $\beta 1_{f i}^{\min }$ yields the minimum reactive power constraint of ISO and also the complementarity constraint $\beta 1_{f i}^{\min }\left(Q_{f i}-Q_{f i}^{\min }\right)=0$. When (18) is differentiated with respect to $\beta 2_{f i}^{\min }$, we obtain $\beta_{f i}^{\min }\left(Q_{f i}-Q_{f i}^{\min }\right)=0$. Here, both $\beta_{f i}^{\min }$ and $\beta 1_{f i}^{\mathrm{min}}$ are matched with the same constraint. All such instances make the NCP nonsquare, which makes these problems harder to solve as compared to the standard Nash games. Leyffer and Munson [13] exploit this redundancy inherent in the NCP formulation to derive nonlinear formulations of the multi-leader-follower games, such as the one posed in this paper. This approach has been used in [12]. This formulation minimizes the complementarity conditions of the EPEC by moving the complementarity conditions into the objective. The remaining constraints are well behaved. The NLP formulation of the EPEC is given as

$$
\begin{aligned}
& \text { Minimize } C_{p e n} \\
& =\left\{\frac{\partial R_{f}}{\partial y^{1}}+\frac{\partial \vec{z}^{I}}{\partial y^{1}} \cdot \vec{w}_{f}^{I}+\frac{\partial \vec{z}^{E}}{\partial y^{1}} \cdot \vec{w}^{E}+\frac{\partial \vec{z}^{C}}{\partial y^{1}} \cdot \vec{w}^{C}\right\}^{T} y^{1} \\
& +\left[\vec{z}^{I}\left(\vec{K}_{1}, \ldots, \vec{K}_{f}, \ldots, \vec{K}_{F}, \mathbf{y}\right)\right]^{T} \vec{w}_{f}^{I} \\
& +\vec{z}^{C}\left(\vec{K}_{1}, \ldots, \vec{K}_{f}, \ldots, \vec{K}_{F}, \mathbf{y}\right) \\
& +\vec{\mu}_{f}^{\min } \cdot\left(\vec{K}_{f}-\vec{K}_{f}^{\min }\right)+\vec{\mu}_{f}^{\max } \cdot\left(\vec{K}_{f}^{\max }-\vec{K}_{f}\right)
\end{aligned}
$$

subject to (19), (20), (22), (24), (26), (27), and (29).

The constraints here do not include any complementarity conditions that have been moved into the objective. It is shown in [13] that the local solution to the above problem with $C_{P e n}=0$ is a strongly stationary point of the multi-leader-follower game. As suggested in [12] and [13], a practical way to solve such problems is to test different initial points. The Nash equilibrium obtained in the examples illustrated in the paper may not be unique. Also, the solutions may form a connected set. As pointed out in [12], a key feature of these equilibria is that they always occur in the field limit of the D-curve of either generator. This may suggest a kind of continuum of equilibria along the $\mathrm{D}$-curve. To some extent, this

$$
\begin{aligned}
L_{f}= & \sum_{i} \rho Q_{f i}-\sum_{i}\left(a_{f i}+b_{f i} Q_{f i}\right) Q_{f i} \\
& +\sum_{i} \mu_{f i}^{\max }\left(k_{f i}^{\max }-k_{f i}\right)+\sum_{i} \mu_{f i}^{\min }\left(k_{f i}-k_{f i}^{\min }\right) \\
& +\sum_{f i} \lambda_{f i}^{o b j}\left(\frac{\partial \ell^{I S O}}{\partial Q_{f i}}\right)+\sum_{i} \lambda_{i}^{v}\left(\frac{\partial \ell^{I S O}}{\partial V_{i}}\right)+\sum_{f i} \lambda_{f i}^{P P}\left(\frac{\partial \ell^{I S O}}{\partial P_{f i}}\right) \\
& +\sum_{i} \lambda_{i}^{\theta}\left(\frac{\partial \ell^{I S O}}{\partial \theta_{i}}\right)+\lambda^{\rho \rho}\left(\frac{\partial \ell^{I S O}}{\partial \rho}\right)+\sum_{i} \lambda 1_{i}^{P}\left(\frac{\partial \ell^{I S O}}{\partial \lambda_{i}^{P}}\right) \\
& +\sum_{i} \lambda 1_{i}^{Q}\left(\frac{\partial \ell^{I S O}}{\partial \lambda_{i}^{Q}}\right)+\sum_{i} \delta 1_{i}^{\min }\left(\frac{\partial \ell^{I S O}}{\partial \delta_{i}^{\min }}\right)+\sum_{i} \delta 1_{i}^{\max }\left(\frac{\partial \ell^{I S O}}{\partial \delta_{i}^{\max }}\right) \\
& +\sum_{f i} \beta 1_{f i}^{\min }\left(\frac{\partial \ell^{I S O}}{\partial \beta_{f i}^{\min }}\right)+\sum_{f i} \beta 1_{f i}^{\max }\left(\frac{\partial \ell^{I S O}}{\partial \beta_{f i}^{\max }}\right)+\sum_{f i} \gamma 1_{f i}\left(\frac{\partial \ell^{I S O}}{\partial \gamma_{f i}}\right) \\
& +\sum_{f i} \alpha 1_{f i}^{\max }\left(\frac{\partial \ell^{I S O}}{\partial \alpha_{f i}^{\max }}\right)+\sum_{f i} \alpha 1_{f i}^{\min }\left(\frac{\partial \ell^{I S O}}{\partial \alpha_{f i}^{\min }}\right) \\
& +\lambda^{\rho \rho 2}\left\{\rho\left(\sum_{f i} Q_{f i}-\sum_{f i} \gamma_{f i}\right)\right\}+\sum_{i} \delta 2_{i}^{\min }\left\{\delta_{i}^{\min }\left(V_{i}-V_{i}^{\min }\right)\right\} \\
& +\sum_{i} \delta 2_{i}^{\max }\left\{\delta_{i}^{\max }\left(V_{i}^{\max }-V_{i}\right)\right\}+\sum_{f i} \beta 2_{f i}^{\min }\left\{\beta_{i}^{\min }\left(Q_{f i}-Q_{f i}^{\min }\right)\right\} \\
& +\sum_{f i} \beta 2_{f i}^{\max }\left\{\beta_{f i}^{\max }\left(Q_{f i}^{\max }-Q_{f i}\right)\right\}+\sum_{f i} \gamma 2_{f i}\left\{\gamma_{i}\left(\rho-k_{f i}\left(a_{f i}+b_{f i} Q_{f i}\right)\right)\right\} \\
& +\sum_{f i} \alpha 2_{f i}^{\min }\left\{\alpha_{i}^{\min }\left(P_{f i}-P_{f i}^{\min }\right)\right\}+\sum_{f i} \alpha 2_{f i}^{\max }\left\{\alpha_{f i}^{\max }\left(P_{f i}^{\max }-P_{f i}\right)\right\} \\
&
\end{aligned}
$$


may come from the trade-off between active and reactive generation that the $\mathrm{D}$-curve introduces. However, this is just the reality of the generation limits. In this paper, the D-curve is simplified to box constraints (constant maximum and minimum levels for reactive power); all these equilibria would not be captured. However, in certain cases, it is found that the equilibrium point shows a kind of symmetry, in terms of reactive power, with respect to the equilibrium where the reactive power levels of gencos are interchanged if the marginal costs of supplying reactive power from these generators are the same.

\section{CAse Study and Discussions}

The multi-leaders (gencos) and single follower (ISO) game is simulated using the NLP formulation of the EPEC model developed in Sections III and IV. The simulations are carried out using the Nordic 32-bus system. The value of the objective function (31), $C_{p e n}$ of all the optimization problems reported in this paper are less than $10^{-9}$; hence, it can be assumed to be sufficiently close to zero. This is required by the theorem reported in [13]. The computational challenges in solving such problems are reported in [12] and [13], so we will not repeat them here. The analysis is carried out to study the impact of strategic behavior of players on reactive power procurement by the ISO and whether the ownership of a generator/synchronous condenser by the ISO can help to mitigate market power. The following cases are considered for analysis:

- Case-A: Competitive market; generators' reactive power price offers are at marginal costs and the market clears at a uniform market price;

- Case-B: Oligopolistic reactive power market with strategic offers from gencos (with $k_{f i}^{\max }=25$ );

- Case-C: Oligopolistic reactive power market with strategic offers from gencos (with $k_{f i}^{\max }=25$ ) in the presence of
ISO-owned generator/synchronous condenser for reactive power provision with a low, pre-declared, reactive power price cap;

- Case-D: All conditions are same as Case-C, except that $k_{f i}^{\max }=5$.

Asymmetry of information on costs prevents the regulator from using a cost plus regulation. As the regulator may not know the cost information of each generator, a standard uniform price cap regulation might be construed as "too tight" by gencos who do not find price caps lucrative enough to supply reactive power, or it is "too loose" from the social benefit viewpoint. A non-intrusive alternative price-cap mechanism is considered in Cases C and D. The models have been developed in GAMS and solved using the CONOPT solver [17]. As explained in [12], the leaders (gencos) problem is nonconvex, and hence, standard NLP solvers can guarantee only a locally optimal solution. The results obtained in the models of strategic behavior (Cases B, C, and D) are simulated using alternative starting values and also by fixing the values of the real and reactive power outputs of the gencos in a narrow range around the solution to the games. This helps in ascertaining whether the profits of the leaders can be improved in a narrow range around the solution to the game or with alternative starting values. The results reported in this paper represent the points for which the results cannot be improved in a reasonable neighborhood ( $\pm 2 \%$ around the reported results).

\section{A. Nordic 32-Bus System}

Let us now consider the 32-bus Nordic power system shown in Fig. 1. There are 20 generators in the system, arbitrarily grouped amongst four gencos. These four gencos along with their associated buses denoting respective generator connections are given below:

- Genco-1: generators at buses 4071, 4072;

$$
\begin{aligned}
\frac{\partial R_{f}}{\partial y^{o}}+\frac{\partial \vec{z}^{I}}{\partial y^{o}} \cdot \vec{w}_{f}^{I}+\frac{\partial \vec{z}^{E}}{\partial y^{o}} \cdot \vec{w}_{f}^{E}+\frac{\partial \vec{z}^{C}}{\partial y^{o}} \cdot \vec{w}_{f}^{C} & =0 \\
\frac{\partial R_{f}}{\partial y^{1}}+\frac{\partial \vec{z}^{I}}{\partial y^{1}} \cdot \vec{w}_{f}^{I}+\frac{\partial \vec{z}^{E}}{\partial y^{1}} \cdot \vec{w}_{f}^{E}+\frac{\partial \vec{z}^{C}}{\partial y^{1}} \cdot \vec{w}_{f}^{C} & \leq 0 \\
\left\{\frac{\partial R_{f}}{\partial y^{1}}+\frac{\partial \vec{z}^{I}}{\partial y^{1}} \cdot \vec{w}_{f}^{I}+\frac{\partial \vec{z}^{E}}{\partial y^{1}} \cdot \vec{w}_{f}^{E}+\frac{\partial \vec{z}^{C}}{\partial y^{1}} \cdot \vec{w}_{f}^{C}\right\}^{T} y^{1} & =0 \\
\vec{z}^{E}\left(\vec{K}_{1}, \ldots, \vec{K}_{f}, \ldots, \vec{K}_{F}, \mathbf{y}\right) & =0 \\
\vec{z}^{C}\left(\vec{K}_{1}, \ldots, \vec{K}_{3}, \ldots, \vec{K}_{F}, \mathbf{y}\right) & =0 \\
\vec{z}^{I}\left(\vec{K}_{1}, \ldots, \vec{K}_{f}, \ldots, \vec{K}_{F}, \mathbf{y}\right) & \geq 0 \\
\frac{\partial R_{f}}{\partial \vec{K}_{f}}+\frac{\partial \vec{z}_{f}^{I}}{\partial \vec{K}_{f}} \cdot \vec{w}_{f}^{I}+\frac{\partial \vec{z}_{f}^{E}}{\partial \vec{K}_{f}} \cdot \vec{w}_{f}^{E}+\frac{\partial \vec{z}_{f}^{C}}{\partial \vec{K}_{f}} \cdot \vec{w}_{f}^{C}+\vec{\mu}_{f}^{\min }-\vec{\mu}_{f}^{\max } & =0 \\
\vec{K}_{f}-\vec{K}_{f}^{\min } & \geq 0 \\
\vec{\mu}_{f}^{\min } \cdot\left(\vec{K}_{f}-\vec{K}_{f}^{\min }\right) & =0, \quad \vec{\mu}_{f}^{\min } \geq 0 \\
\vec{K}_{f}^{\max }-\vec{K}_{f} & \geq 0 \\
\vec{\mu}_{f}^{\max } \cdot\left(\vec{K}_{f}^{\max }-\vec{K}_{f}\right) & =0, \quad \vec{\mu}_{f}^{\max } \geq 0
\end{aligned}
$$




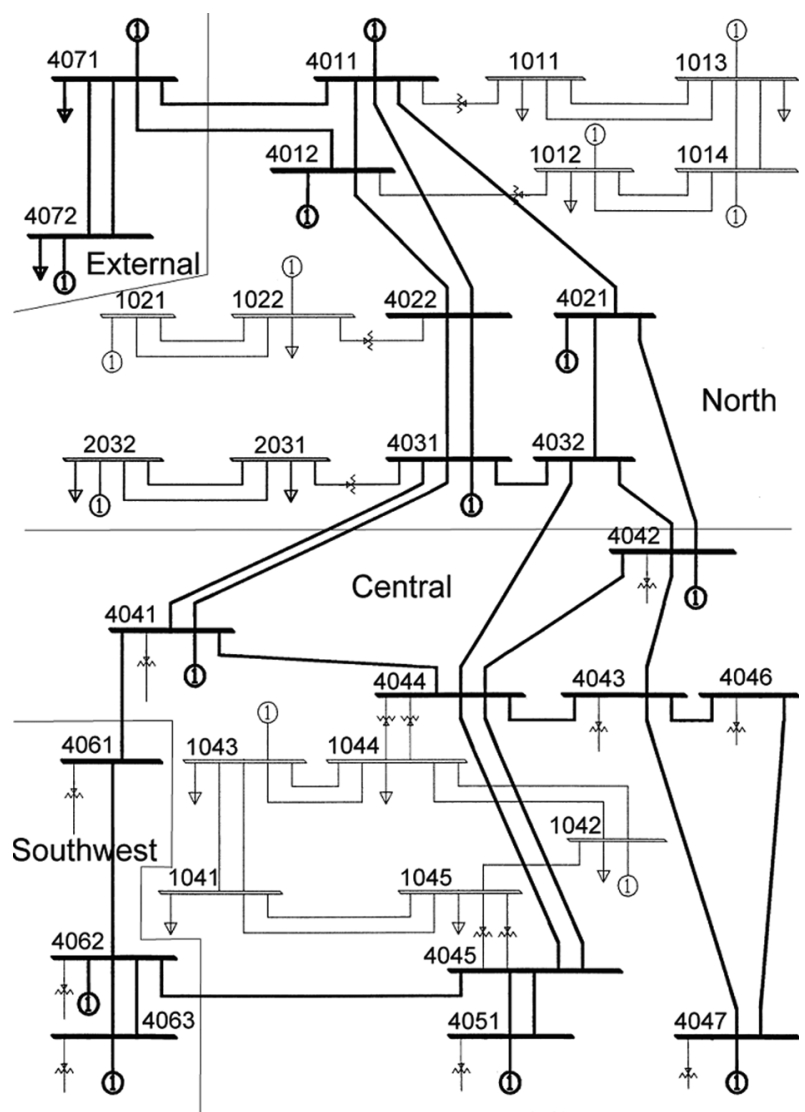

Fig. 1. Nordic 32-bus system.

- Genco-2: generators at buses 4011, 4012, 4021, 4031, 2032, 1012, 1013, 1014, 1021, and 1022;

- Genco-3: generators at buses 4042, 4041, 4051, 4057, 1043, and 1042;

- Genco-4: generators at buses 4062 and 4063.

All the generators can provide both active and reactive power, but as mentioned earlier, it is assumed that the active power dispatch has already been carried out. The reactive power generation characteristics are given in Table I.

A computational challenge is the determination of the initial points which lead the objective function of the NLP formulation to near zero (less than $10^{-9}$ ) of the proposed MPEC. The simulation results are provided in Tables II-IV.

\section{B. Discussions}

1) Strategic Behavior in the System: Comparing the total payment for reactive power in Cases A and B (Table II), we find that the total payment in case of the perfect competition with uniform market price (Case A) is $\$ 3.644 / \mathrm{h}$, while in the case of strategic offers is $\$ 71.58 / \mathrm{h}$ (Case B). In Case B, we allow the upper limit on strategic offer parameter $k_{f i}^{\max }$ to be a very large number, $k_{f i}^{\max }=25$. This is confirmed from column 3 of Table III, where it is observed that most generators set their offer strategy $\left(k_{f i}\right)$ as high as possible, which is 25 . Such a strategic behavior is understandable and it increases the ISO's payment burden for reactive power significantly.

It is interesting to note that the reactive power output of the generators increases at bus 1012 and 1014 in case B (Table IV).
TABLE I

GENERATOR DATA FOR GENERATORS IN THE NORDIC SYSTEM

\begin{tabular}{|c|c|c|c|}
\hline Bus \# & $Q^{\max }(\mathrm{pu})$ & $a_{f i}$ & $b_{f i}$ \\
\hline 4072 & 10 & 1.25 & 0.06 \\
\hline 4071 & 2.50 & 0.1 & 0.07 \\
\hline 4011 & 5.00 & 0.24 & 0.09 \\
\hline 4012 & 4.00 & 0.67 & 0.04 \\
\hline 4021 & 1.50 & 0.72 & 0.07 \\
\hline 4031 & 1.75 & 0.61 & 0.05 \\
\hline 4042 & 3.50 & 1.7 & 0.07 \\
\hline 4041 & 3.00 & 1.03 & 0.073 \\
\hline 4062 & 3.00 & 0.4 & 0.05 \\
\hline 4063 & 6.00 & 0.86 & 0.05 \\
\hline 4051 & 3.50 & 0.37 & 0.09 \\
\hline 4047 & 6.00 & 0.75 & 0.05 \\
\hline 2032 & 4.25 & 0.89 & 0.02 \\
\hline 1013 & 3.00 & 0.63 & 0.06 \\
\hline 1012 & 4.00 & 0.92 & 0.03 \\
\hline 1014 & 3.50 & 0.2 & 0.05 \\
\hline 1022 & 1.25 & 1.58 & 0.04 \\
\hline 1021 & 3.00 & 0.32 & 0.04 \\
\hline 1043 & 1.00 & 0.68 & 0.05 \\
\hline 1042 & 2.00 & 0.65 & 0.05 \\
\hline
\end{tabular}

TABLE II

COMPARISON OF UNIFORM PRICES AND TOTAL PAYMENT

\begin{tabular}{|c|c|c|c|c|}
\hline & Case A & Case B & Case C & Case D \\
\hline$\rho(\$ /$ pu MVAr) & 1.7 & 33.27 & 8 & 1.85 \\
\hline Total payment $(\$ / \mathrm{hr})$ & 3.644 & 71.58 & 17.15 & 3.981 \\
\hline
\end{tabular}

TABLE III

COMPARISON OF THE STRATEGIC VARIABLES, REVENUES AND PROFITS

\begin{tabular}{|c|c|c|c|c|c|c|c|c|c|c|c|c|}
\hline \multirow{3}{*}{ Genco } & \multirow{3}{*}{ Bus } & \multicolumn{3}{|c|}{$k_{f i}$} & \multicolumn{4}{|c|}{ Revenue (\$/hr) } & \multicolumn{4}{|c|}{ Profit (\$/hr) } \\
\hline & & \multicolumn{3}{|c|}{ Case } & \multicolumn{4}{|c|}{ Case } & \multicolumn{4}{|c|}{ Case } \\
\hline & & B & $\mathrm{C}$ & D & A & B & $\mathrm{C}$ & $\mathrm{D}$ & A & B & $\mathrm{C}$ & $\mathrm{D}$ \\
\hline 1 & $\begin{array}{l}4072 \\
4071 \\
\end{array}$ & $\begin{array}{c}25 \\
21.12\end{array}$ & \begin{tabular}{|c|}
6.08 \\
18.91 \\
\end{tabular} & $\begin{array}{l}1.46 \\
2.92 \\
\end{array}$ & 2.66 & 44.7 & 4.75 & 0.64 & 0.6 & 42.9 & 3.98 & 0.20 \\
\hline 2 & $\begin{array}{l}4011 \\
4012 \\
4021 \\
4031 \\
2032 \\
1013 \\
1012 \\
1014 \\
1022 \\
1021\end{array}$ & \begin{tabular}{||c}
12.99 \\
25 \\
25 \\
25 \\
25 \\
25 \\
25 \\
20.98 \\
21.05 \\
25
\end{tabular} & \begin{tabular}{|c|}
25 \\
11.94 \\
11.11 \\
13.12 \\
8.98 \\
12.69 \\
8.70 \\
14.39 \\
5.06 \\
25
\end{tabular} & $\begin{array}{l}5.00 \\
2.76 \\
2.57 \\
3.03 \\
2.08 \\
2.94 \\
1.99 \\
3.22 \\
1.17 \\
3.23\end{array}$ & 0.05 & 8.66 & 0 & 0.48 & .02 & 8.42 & 0 & 0.24 \\
\hline 3 & $\begin{array}{l}4042 \\
4041 \\
4051 \\
4047 \\
1043 \\
1042 \\
\end{array}$ & \begin{tabular}{||c}
19.31 \\
25 \\
25 \\
25 \\
25 \\
25 \\
\end{tabular} & \begin{tabular}{|c|}
4.71 \\
7.77 \\
21.62 \\
10.67 \\
11.77 \\
12.31 \\
\end{tabular} & $\begin{array}{l}1.09 \\
1.80 \\
5.00 \\
2.47 \\
2.72 \\
2.85 \\
\end{array}$ & 0 & 0 & 0 & 0 & 0 & 0 & 0 & 0 \\
\hline 4 & $\begin{array}{l}4062 \\
4063\end{array}$ & $\begin{array}{l}25 \\
25\end{array}$ & \begin{tabular}{|l}
19.97 \\
9.014
\end{tabular} & $\begin{array}{l}4.63 \\
2.09\end{array}$ & 0.93 & 18.2 & 4.41 & 1.01 & .45 & 17.7 & 3.92 & 0.53 \\
\hline D-owne & 4072 & - & 1 & 1 & - & - & 8 & 1.85 & - & - & 7.05 & 0.90 \\
\hline
\end{tabular}

This is counter-intuitive to the notion that firms withhold output in oligopolistic markets. The generators may increase their reactive power output in case they observe that doing so allows them to increase their profit maximizing levels without compromising the network reliability. This is due to the local market power of generators in reactive power market.

2) Alternative Price Cap Regulation: In Case C, which presents the alternative regulation, the ISO sets up a generator 
TABLE IV

COMPARISON OF P AND Q IN Four CASES $\left(S_{\text {base }}=100 \mathrm{MVA}\right)$

\begin{tabular}{|c|c|c|c|c|c|c|c|c|}
\hline \multirow{2}{*}{ BUS \# } & \multicolumn{4}{|c|}{$\mathrm{P}(\mathrm{pu})$} & \multicolumn{4}{|c|}{$\mathrm{Q}(\mathrm{pu})$} \\
\hline & Case A & Case B & Case C & Case D & Case A & Case B & Case $\mathrm{C}$ & Case D \\
\hline 4072 & 20.15 & 20.15 & 20.15 & 20.15 & 1.57 & 1.34 & $\begin{array}{l}0.593+ \\
1.0 \text { (SO) }\end{array}$ & $\begin{array}{c}0.344+ \\
1.0 \text { (SO) }\end{array}$ \\
\hline 4071 & 4.81 & 4.72 & 4.82 & 4.72 & & & & \\
\hline 4011 & 10 & 10 & 10 & 10 & . & & . & . \\
\hline 4012 & 8 & 8 & 8 & 8 & . & . & . & . \\
\hline 4021 & 3 & 3 & 3 & 3 & . & . & $\cdot$ & r \\
\hline 4031 & 3.5 & 3.5 & 3.5 & 3.5 & . & • & . & . \\
\hline 2032 & 7.14 & 7.15 & 7.14 & 7.15 & . & & . & . \\
\hline 1013 & 1.95 & 2.23 & 1.95 & 2.24 & & & . & . \\
\hline 1012 & 5.27 & 4.95 & 5.27 & 4.95 & 0.027 & 0.258 & . & 0.258 \\
\hline 1014 & 0.7 & 0.7 & 0.7 & 0.7 & & 0.002 & . & 0.002 \\
\hline 1022 & 2.5 & 2.5 & 2.5 & 2.5 & & & . & . \\
\hline 1021 & 1.67 & 1.66 & 1.67 & 1.66 & & & & \\
\hline 4042 & 4.52 & 4.67 & 4.49 & 4.67 & & & . & \\
\hline 4041 & 0 & 0 & 0 & 0 & . & & . & . \\
\hline 4051 & 7 & 7 & 7 & 7 & . & & . & . \\
\hline 4047 & 12 & 12 & 12 & 12 & . & & . & . \\
\hline 1043 & 1.84 & 1.78 & 1.85 & 1.78 & & & . & . \\
\hline 1042 & 4 & 4 & 4. & 4 & & & & \\
\hline 4062 & 6 & 6 & 6 & 6 & & & . & . \\
\hline 4063 & 9.28 & 9.30 & 9.28 & 9.30 & 0.55 & 0.548 & 0.551 & 0.548 \\
\hline Total & 113.31 & 113.30 & 113.31 & 113.30 & \begin{tabular}{|l|}
2.144 \\
\end{tabular} & 2.152 & 2.144 & 2.152 \\
\hline
\end{tabular}

or synchronous condenser owned by itself at a strategic bus and declares a low value of $k_{I S O, i}^{\max }$ for that unit. The subscript ISO denotes the ownership of the unit by the ISO. All strategic generators take into consideration the low value of $k_{I S O, i}^{\max }$ in their respective optimization problems, re-strategize, and change their offers to maximize their profits. The proposed alternative regulatory approach is commonly termed as "Yardstick Regulation", which has been applied in the electricity markets of South America [18].

The idea of intervention by the ISO is to create conditions so that the competing gencos offer to supply as close as possible to their marginal costs. Hence, it is important to know where to install the ISO owned unit. This is important because a suboptimal selection of location and capacity by the ISO could have adverse impacts on grid operation and the price. Application of alternative price cap regulation proposed in this paper applied to a large network is a challenging problem, not only because of the associated numerical convergence issues but also because of the complexities involved in the selection of the bus where the ISO should locate its generator or condenser.

In this paper, the results reported in [8] are used, where must-run indices are used to identify the bus with maximum must-run capacity in reactive power. In the system under consideration, the generator at bus 4072 has considerable market power. This was also reported in earlier research [6], [7]. Now, if the ISO owns a synchronous condenser of $100 \mathrm{MVAr}$ capacity at bus-4072 and considering $k_{I S O, 4072}^{\max }=1$, it is seen that the uniform market price decreases to $\$ 8 /$ p.u.MVAr against \$33.27/p.u.MVAr (Case B) (Table II). This is because other generators must choose $k_{f i}$ and the corresponding $Q_{f i}$ to maximize their profits subject to satisfying the constraint $k_{f i}\left(a_{f i}+b_{f i} Q_{f i}\right) \leq \rho$. Therefore, they find that there is a trade-off between increasing $k_{f i}$ and $Q_{f i}$. The presence of ISO-owned synchronous condenser, which unilaterally declares a lower cap on strategic variable, therefore forces the competing gencos in Case $\mathrm{C}$ to lower their strategic variable as shown in column 4 of Table III.

As stated earlier in this section, the intervention by the ISO seeks to mimic the conditions of competition. In Case $\mathrm{C}$, the ISO might view that uniform market price equal to $\$ 8 /$ p.u. MVAr is still high as compared to a competitive market (Case A). In such conditions, the ISO, along with the presence of ISOowned synchronous condenser with a pre-declared $k_{I S O, 4072}^{\max }=$ 1 , might place an arbitrary cap (depending on the minimum level that will induce the gencos to supply reactive power) on the bids. In this paper in Case D, we assume that the ISO has a rough estimate of the cost based on the generation technology of generator (type of generators) at each node and hence sets a cap equal to five times the estimated marginal cost at each node, i.e., $k_{f i}^{\max }=5$. In Case D, we observe from Table II that this combination of price cap and alternative price cap mechanism, proposed in this paper, leads to a uniform market price of $1.85 \$ /$ p.u. MVAr, which is then reasonably close to the uniform market price in Case A.

It should be noted that in Case $\mathrm{C}$, the values of $k_{f i}$ of the generators are still much higher than unity, and the market price and payments are still much higher than Case A. This problem is because of the local nature of reactive power supply. Exercise of market power cannot be completely avoided. Reactive power generated by ISO-owned synchronous condenser cannot be transmitted over long distances as a "strategic supply" for reactive power demand at distant buses. Nevertheless, this problem can be minimized by selecting multiple nodes for location of ISO owned synchronous condensers in large power systems or by using a combination or price cap regulation and alternative price cap regulation as demonstrated in Case D.

\section{CONCLUding REMARKS}

In this paper, the strategic behavior of generators and synchronous condensers in the reactive power market is examined using the supply function equilibrium framework based on nonlinear ac load flow. Because of the local nature of reactive power supply, it is observed that the equilibrium between the suppliers of reactive power settles very close to the price-cap in most cases. Also, if a genco has reactive power supply sources at multiple locations, it can strategically act to sometimes supply more reactive power. Changing the price-cap in either direction could be construed as either allowing the suppliers too much or not giving them adequate incentives to participate in the market. An alternative price-cap regulatory mechanism is proposed and tested, where the participation by the ISO owned supply of reactive power with pre-declared lower price-cap helps mitigate some of the shortcomings of gaming by private generators.

\section{APPENDIX}

The KKT conditions of the SO's problem are in (A1)-(A22) on the following two pages. 


$$
\begin{aligned}
& \frac{\partial \ell^{I S O}}{\partial Q_{f i}}=\rho+\lambda_{i}^{Q}-\beta_{f i}^{\min }+\beta_{f i}^{\max }+k_{f i} b_{f i} \gamma_{f i}=0 \quad \forall f i \\
& \frac{\partial \ell^{I S O}}{\partial V_{i}}=\lambda_{i}^{P}\left(-2 V_{i} G_{i i}-\sum_{j \neq i} V_{j}\left(G_{i j} \cos \left(\theta_{i}-\theta_{j}\right)+B_{i j} \sin \left(\theta_{i}-\theta_{j}\right)\right)\right) \\
& -\sum_{j \neq i} \lambda_{j}^{P} V_{j}\left(G_{j i} \cos \left(\theta_{j}-\theta_{i}\right)+B_{j i} \sin \left(\theta_{j}-\theta_{i}\right)\right) \\
& +\lambda_{i}^{Q}\left(2 V_{i} B_{i i}-\sum_{j \neq i} V_{j}\left(G_{i j} \sin \left(\theta_{i}-\theta_{j}\right)-B_{i j} \cos \left(\theta_{i}-\theta_{j}\right)\right)\right) \\
& -\sum_{j \neq i} \lambda_{j}^{Q} V_{j}\left(G_{j i} \sin \left(\theta_{j}-\theta_{i}\right)-B_{j i} \cos \left(\theta_{j}-\theta_{i}\right)\right)-\delta_{i}^{\min }+\delta_{i}^{\max }=0 \quad \forall i \\
& \frac{\partial \ell^{I S O}}{\partial P_{f i}}=\lambda_{i}^{P}-\alpha_{f i}^{\min }+\alpha_{f i}^{\max }=0 \quad \forall f i \\
& \left.\frac{\partial \ell^{I S O}}{\partial \theta_{i}}=\lambda_{i}^{P} \sum_{j \neq i} V_{i} V_{j}\left(-G_{i j} \sin \left(\theta_{i}-\theta_{j}\right)+B_{i j} \sin \left(\theta_{i}-\theta_{j}\right)\right)\right) \\
& -\sum_{j \neq i} \lambda_{j}^{P} V_{i} V_{j}\left(G_{j i} \sin \left(\theta_{j}-\theta_{i}\right)-B_{j i} \cos \left(\theta_{j}-\theta_{i}\right)\right) \\
& -\lambda_{i}^{Q}\left(\sum_{j \neq i} V_{i} V_{j}\left(G_{i j} \cos \left(\theta_{i}-\theta_{j}\right)+B_{i j} \sin \left(\theta_{i}-\theta_{j}\right)\right)\right) \\
& -\sum_{j \neq i} \lambda_{j}^{Q} V_{j} V_{i}\left(-G_{j i} \cos \left(\theta_{j}-\theta_{i}\right)+B_{j i} \sin \left(\theta_{j}-\theta_{i}\right)\right)=0 \forall i \\
& \frac{\partial \ell^{I S O}}{\partial \rho}=\sum_{f i} Q_{f i}-\sum_{f i} \gamma_{f i} \geq 0 \\
& \rho\left(\sum_{f i} Q_{f i}-\sum_{f i} \gamma_{f i}\right)=0, \rho \geq 0 \\
& \frac{\partial \ell^{I S O}}{\partial \lambda_{i}^{P}}=\sum_{f} P_{f i}-P D_{i} \\
& -\sum_{j} V_{i} V_{j}\left(G_{i j} \cos \left(\theta_{i}-\theta_{j}\right)+B_{i j} \sin \left(\theta_{i}-\theta_{j}\right)\right)=0 \forall i \\
& \frac{\partial \ell^{I S O}}{\partial \lambda_{i}^{Q}}=\sum_{f} Q_{f i}-Q D_{i} \\
& -\sum_{j} V_{i} V_{j}\left(G_{i j} \sin \left(\theta_{i}-\theta_{j}\right)-B_{i j} \cos \left(\theta_{i}-\theta_{j}\right)\right)=0 \forall i \\
& \frac{\partial \ell^{I S O}}{\partial \delta_{i}^{\min }}=-V_{i}+V_{i}^{\min } \leq 0, \forall i \\
& \delta_{i}^{\min }\left(-V_{i}+V_{i}^{\min }\right)=0, \delta_{i}^{\min } \geq 0 \forall i \\
& \frac{\partial \ell^{I S O}}{\partial \delta_{i}^{\max }}=V_{i}-V_{i}^{\max } \leq 0, \forall i \\
& \delta_{i}^{\max }\left(V_{i}-V_{i}^{\max }\right)=0, \delta_{i}^{\max } \geq 0 \forall i \\
& \frac{\partial \ell^{I S O}}{\partial \beta_{f i}^{\min }}=-Q_{f i}+Q_{f i}^{\min } \leq 0, \forall f i \\
& \beta_{f i}^{\min }\left(-Q_{f i}+Q_{f i}^{\min }\right)=0, \beta_{f i}^{\min } \geq 0 \forall f i \\
& \frac{\partial \ell^{I S O}}{\partial \beta_{f i}^{\max }}=Q_{f i}-Q_{f i}^{\max } \leq 0, \forall f i \\
& \beta_{f i}^{\max }\left(Q_{f i}-Q_{f i}^{\max }\right)=0, \beta_{f i}^{\max } \geq 0 \forall f i
\end{aligned}
$$




$$
\begin{aligned}
& \frac{\partial \ell^{I S O}}{\partial \gamma_{f i}}=k_{f i}\left(a_{f i}+b_{f i} Q_{f i}\right)-\rho \leq 0, \forall f i \\
& \gamma_{f i}\left(k_{f i}\left(a_{f i}+b_{f i} Q_{f i}\right)-\rho\right)=0, \gamma_{f i} \geq 0 \forall f i \\
& \frac{\partial \ell^{I S O}}{\partial \alpha_{f i}^{\min }}=-P_{f i}+P_{f i}^{\min } \leq 0, \forall f i \\
& \alpha_{f i}^{\min }\left(-P_{f i}+P_{f i}^{\min }\right)=0, \alpha_{f i}^{\min } \geq 0 \forall f i \\
& \frac{\partial \ell^{I S O}}{\partial \alpha_{f i}^{\max }}=P_{f i}-P_{f i}^{\max } \leq 0, \forall f i \\
& \alpha_{f i}^{\max }\left(P_{f i}-P_{f i}^{\max }\right)=0, \alpha_{f i}^{\max } \geq 0 \forall f i
\end{aligned}
$$

\section{REFERENCES}

[1] FERC Staff Report, Principles for Efficient and Reliable Reactive Power Supply and Consumption, 2005.

[2] I. El-Samahy, K. Bhattacharya, C. Canizares, M. Anjos, and J. Pan, "A procurement market model for reactive power services considering system security," IEEE Trans. Power Syst., vol. 23, no. 1, pp. 137-149, Feb. 2008

[3] S. Ahmed and G. Strbac, "A method for simulation and analysis of reactive power market," IEEE Trans. Power Syst., vol. 15, no. 3, pp. 1047-1052, Aug. 2000.

[4] J. B. Gil, T. G. S. Roman, J. J. A. Rios, and P. S. Martin, "Reactive power pricing: a conceptual framework for remuneration and charging procedures," IEEE Trans. Power Syst., vol. 15, no. 2, pp. 483-489, May 2000.

[5] K. Bhattacharya and J. Zhong, "Reactive power as an ancillary service," IEEE Trans. Power Syst., vol. 16, no. 2, pp. 294-300, May 2001.

[6] J. Zhong and K. Bhattacharya, "Toward a competitive market for reactive power," IEEE Trans. Power Syst., vol. 17, no. 4, pp. 1206-1215, Nov. 2002.

[7] J. Zhong, E. Nobile, A. Bose, and K. Bhattacharya, "Localized reactive power markets using the concept of voltage control areas," IEEE Trans. Power Syst., vol. 19, no. 3, pp. 1555-1561, Aug. 2004.

[8] D. Feng, J. Zhong, and D. Gan, "Reactive market power analysis using must run indices," IEEE Trans. Power Syst., vol. 23, no. 2, pp. 755-765, May 2008.

[9] B. F. Hobbs, "Linear complementarity models of Nash-Cournot competition in bilateral and POOLCO power markets," IEEE Trans. Power Syst., vol. 16, no. 2, pp. 194-202, May 2001.

[10] C. J. Day, B. F. Hobbs, and J. S. Pang, "Oligopilistic competition in power networks: A conjectured supply function approach," IEEE Trans. Power Syst., vol. 17, no. 3, pp. 597-607, Aug. 2002.

[11] X. Wang, Y. Li, and S. Zhang, "Oligopolistic equilibrium analysis for electricity markets: A nonlinear complementarity approach," IEEE Trans. Power Syst., vol. 19, no. 3, pp. 1348-1355, Aug. 2004.

[12] G. Bautista, M. F. Anjos, and A. Vannelli, "Formulation of oligopolistic competition in AC power networks: An NLP approach," IEEE Trans. Power Syst., vol. 22, no. 1, pp. 105-115, Feb. 2007.

[13] S. Leyffer and T. Munson, "Solving multi-leader-follower games," Preprint Argonne National Laboratory, vol. ANL/MCS-P1243-0405, Apr. 2005.

[14] R. Baldick, "Electricity market equilibrium models: The effect of parameterization," IEEE Trans. Power Syst., vol. 17, pp. 1170-1176, 2002.

[15] J. S. Pang and M. Fukushima, Quasi-Variational Inequalities, Generalized Nash Equilibria, and Multi-Leader-Follower Games, John Hopkins Univ., Baltimore, MD, 2002.
[16] A. Ehrenmann and K. Neuhoff, "A comparison of electricity market design in networks," in Cambridge Working Papers in Economics. Cambridge, U.K.: Dept. Appl. Econ., Univ. Cambridge, 2004.

[17] GAMS Release 2.50, A user's guide, GAMS Development Corporation, 2006.

[18] H. Rudnick and J. A. Donoso, "Integration of price cap and yardstick competition schemes in electrical distribution regulation," IEEE Trans. Power Syst., vol. 15, no. 4, pp. 1428-1433, Nov. 2000.

Puneet Chitkara (M'08) received the B.E. degree in electrical engineering from Maulana Azad National Institute of Technology, Bhopal, India, in 1993 and the Ph.D. degree from Indira Gandhi Institute of Development Research, Mumbai, India, in 1999.

At present, he is a Senior Research Assistant in the Department of Electrical and Electronic Engineering, The University of Hong Kong. His areas of interest are regulatory economics, applications of game theory in power markets, and electricity sector deregulation.

Jin Zhong (S'00-M'04) received the B.Sc. degree from Tsinghua University, Beijing, China, in 1995, the M.Sc. degree from China Electric Power Research Institute, Beijing, in 1998, and the Ph.D. degree from Chalmers University of Technology, Gothenburg, Sweden, in 2003.

At present, she is an Assistant Professor in the Department of Electrical and Electronic Engineering, The University of Hong Kong. Her areas of interest are electricity sector deregulation, ancillary service pricing, and power system planning.

Kankar Bhattacharya (M'95-SM'01) received the $\mathrm{Ph} . \mathrm{D}$. degree in electrical engineering from Indian Institute of Technology, New Delhi, India, in 1993.

He was in the faculty of Indira Gandhi Institute of Development Research, Mumbai, India, during 1993-1998, and the Department of Electric Power Engineering, Chalmers University of Technology, Gothenburg, Sweden, during 1998-2002. Since January 2003, he has been with the Department of Electrical and Computer Engineering, University of Waterloo, Waterloo, ON, Canada, and currently he is a Professor. His research interests are in power system dynamics, stability and control, economic operations planning, electricity pricing, and electric utility deregulation. 